genitalium-positive NGU. The purpose of this study was to establish the treatment efficacy of sitafloxacin (STFX), one of the new generation of quinolones, for patients with NGU.

Male patients with NGU were included in this study. Chlamydia trachomatis was detected by TMA assay and $\mathrm{M}$. genitalium and Ureaplasma urealyticum were detected by real-time PCR. The patients received STFX 100mg twice daily for 7 days orally. The primary outcome was microbiological eradication at 2 to 4 weeks after completion of treatment.

A total of 208 patients were initially included in this study; however, 18 who were Neisseria gonorrhoeae-positive, 36 who failed to visit again, 34 who visited within 2 weeks after completion of treatment, 1 who had sexual intercourse with his female partner, and 1 whose data was lost were excluded from further analysis. In the 118 patients who could be analysed, the microbiological eradication rates were $95.7 \%$ (45/47) for C. trachomatis, 93.8\% (15/16) for M. genitalium, and $100 \%$ (17/17) for U. urealyticum.

The results of this study clearly show that STFX has strong activity against $C$. trachomatis, $M$. genitalium and $U$. urealyticum, which are common pathogens of NGU. The regimen with STFX for patients with NGU should be recommended as a standard one.

\section{P2.101 HIV STATUS AND OTHER PREDICTORS OF SUCCESSFUL SYPHILIS TREATMENT}

doi:10.1136/sextrans-2013-051184.0365

R P Kohn, K Bernstein, S Cohen, M Chen, S Philip. San Francisco Department of Public Health, San Francisco, CA, United States

Background Data addressing the question of whether HIV-positive patients respond as well to the recommended treatment for syphilis as HIV-negative patients are sparse. We examined data from reported early syphilis cases in San Francisco to identify factors related to serologic response to treatment.

Methods San Francisco early syphilis cases diagnosed between 2006 and 2012 were analysed in terms of serologic response to treatment. Cases were excluded if the patient had any prior syphilis diagnosis reported, and only cases with an initial reactive serologic test for syphilis (STS) titer of 1:4 or higher were included. A successful serologic response to treatment was defined by a record of a nonreactive STS or a four-fold titer decrease within 12 months from treatment. Survival analysis and proportional hazards models were used to examine the relationship between demographic and risk factor data, including HIV status, and number of days until successful serologic response was documented.

Results A total of 1664 first-time cases were examined. HIV-positive patients were significantly more likely to have a follow-up STS than other patients $(p<0.0001)$. Of the 1557 cases with any followup STS, 9.3 percent did not show evidence of successful serologic response. HIV-negative patients were not found to be less likely to show response to treatment (median of 111 days for HIV-positives versus 124.5 for HIV-negatives, $\mathrm{p}<0.0001$ ). Stage of disease was also associated with evidence of serologic response (median of 121.0 days for primary, 109.0 days for secondary, and 130.0 for early latent), but race, gender, genders of partners, and treatment provided were not.

Conclusion Analysis of routine interview data found no evidence that HIV-positive patients failed to respond to standard syphilis treatments. However, the limits of surveillance data suggest the need for further research examining the relationship between immune status and response to treatment among HIV-positive patients.

\section{P2.102 THE INFLUENCE OF ANTI RETROVIRAL TREATMENT (ART) ON THE TREATMENT OF TRICHOMONAS VAGINALIS AMONG HIV-INFECTED WOMEN IN THREE SOUTHERN CITIES THE U.S.}

doi:10.1136/sextrans-2013-051184.0366
P Kissinger, ${ }^{1} \mathrm{~A}$ Adamski, ${ }^{2} \mathrm{R}$ A Clark, ${ }^{3} \mathrm{~L}$ Mena, ${ }^{3} \mathrm{H}$ Henderson, ${ }^{4} \mathrm{~J}$ Levison, ${ }^{1} \mathrm{~N}$ Schmidt, 2D Martin. 'Tulane University SPHTM, New Orleans, LA, United States; 'Louisiana State University Health Sciences Center, New Orleans, LA, United States; ${ }^{3}$ University of Mississippi - Department of Medicine, Jackson, MS, United States; ${ }^{4}$ Baylor College of Medicine, Houston, TX, United States

Background Trichomonas vaginalis (TV) is the most common non-viral STI and has been linked to premature membrane rupture, preterm birth, and low birth weight in pregnant women. TV has also been shown to increase vaginal shedding of HIV and, thus, may influence HIV sexual and perinatal transmission. Repeat infection rates among HIV + women are high. We have shown that bacterial vaginosis $(\mathrm{BV})$ is associated with single dose metronidazole (MTZ) treatment failure in HIV+ women. A recent study in Africa has found that nevirapine is also associated with a higher rate of repeat infections. The purpose of this study is to determine if other ART interferes with single dose MTZ treatment of TV.

Methods A secondary data analysis was performed on a cohort of $\mathrm{HIV}+/ \mathrm{TV}+$ women who had been randomised to single $(2 \mathrm{gm})$ dose or 7 day (500mg BID) dose MTZ. Follow-up visit, including culture, occurred 6-12 days after treatment completion. Data on sexual exposure was collected. Repeat TV infection rates were compared for women on ART at baseline versus not on ART, controlling for $\mathrm{BV}$ and treatment arm.

Results Of the 230 women included, $65 \%$ were receiving ART: NRTI (95\%), NNRTI (31\%), PI (58\%) and other ART (2\%). Those on ART had higher repeat infections than women not on ART [25/150 (16.7\%) vs. 6/80 (6.3\%), p-value $=0.03]$. Controlling for BV status and stratifying by treatment arm, the association was found only in the single-dose arm ( $p$-value $=0.05)$ and not in the multidose arm ( $p$-value $=0.39)$. Only $5 \%$ of the women were sexually re-exposed during follow-up.

Conclusions ART in general is associated with a higher TV repeat infection rate following single dose MTZ treatment but not for multi-dose. These data further support the recommendation that single dose MTZ not be used in HIV + women

\section{P2.103 DIFFERENTIAL EFFECT OF STANDARD THERAPIES FOR NON-GONOCOCCAL URETHRITIS AGAINST UREAPLASMA SPECIES}

doi:10.1136/sextrans-2013-051184.0367

${ }^{1}$ C M Khosropour, 'L E Manhart, ${ }^{2} \mathrm{C}$ W Gillespie, ${ }^{3} \mathrm{M}$ Lowens, ${ }^{1,3} \mathrm{M}$ R Golden, ${ }^{1} \mathrm{P}$ A Totten. 'University of Washington, Seattle, WA, United States; ${ }^{2}$ Children's National Medical Center, Washington, DC, United States; ${ }^{3}$ STD Program, Public Health - Seattle and King County, Seattle, WA, United States

Background U. urealyticum (UU) but not U. parvum (UP) is associated with non-gonococcal urethritis (NGU), while UP may be associated with adverse pregnancy outcomes. Treatment failure may differ by species; therefore, we examined the efficacy of CDC-recommended therapies for NGU against UU and UP separately.

Methods From May 2007 to July 2011, men aged $\geq 16$ years attending an STD clinic in Seattle, Washington with NGU (urethral discharge or urethral symptoms plus $\geq 5 \mathrm{PMNs} / \mathrm{HPF}$ ) were enrolled in a randomised treatment trial. Participants received active azithromy$\operatorname{cin}(1 \mathrm{~g})$ and placebo doxycycline or active doxycycline $(100 \mathrm{mg}$ bid $\times$ 7d) and placebo azithromycin. Ureaplasma species were detected in broth urine culture followed by species-specific PCR. Microbiologic failure (detected by PCR) was determined at 3, 6, and 9 weeks. At 3 weeks, men who failed initial treatment received the alternate therapy (active doxycycline if they first received active azithromycin and vice versa). Persistent failures received moxifloxacin at 6 weeks.

Results Of 479 enrolled men, 107 (22.3\%) and 59 (12.3\%) were infected with UU and UP, respectively, and returned at 3 weeks. Among men who received azithromycin, microbiologic failure at 3 weeks occurred in $46.7 \%(14 / 30)$ of UP-infected men and $25.0 \%$ 